\title{
Metodologia de avaliação do trabalho na atenção primária à saúde
}

\author{
Methodology for assessment of activities \\ in primary health care
}

Arnaldo Sala 1

Maria Ines Baptistella Nemes 1

Diane Dede Cohen 1

\footnotetext{
1 Departamento de Medicina Preventiva, Faculdade de Medicina, Universidade de São Paulo. Centro de Saúde Escola Prof. Samuel B. Pessoa. Av. Vital Brasil 1490, São Paulo, SP 05503-000, Brasil. arnasala@usp.br
}

A bstract A case study assessed health care activities in a primary health care facility to help devel op methodol ogies of care work assessment and of the proposal of leading categories for the said processes, based on the work process. This study was devel oped at the Centro de Saúde Escola of the Faculdade de Medicina of the Universi dade de São Paulo, using as empirical material the assessment of the care acti vities aimed at the emergency care and programmed care of 'chronic' pati ents ( pati ents suffering from hypertension and diabetes). The analysis of these assessments which considered different moments of the work process (working objects, activity of agents, analysis of the working means and result of the work) suggest the need of creating anaIytical categories that will make workable the understanding of this process, inserting each moment to the work as a whole. The analytical categories proposed are: 1) workability as a means of making effective the proposed actions; 2) strategic effectiveness as a measure of the scope of the results that have been observed among patients and/or the population and that can be the result of the actions performed.

Key words Hypertension; Primary Health Care; Ambulatory Care; Outcome and Process Assessment (Health Care)

Resumo No presente trabalho, realiza-se um estudo de caso, no qual se analisa o processo de avaliação de ações assistenciais em uma unidade básica de saúde, no sentido de contribuir para o desenvolvimento de metodologias de avaliação do trabal ho assistencial e de proposição de categorias ori entadoras desses processos de avaliação, tendo como base o processo de trabalho. Este estudo foi desenvol vido no Centro de Saúde Escola da Facul dade de Medicina da Universidade de São Paulo, tomando como seu material empírico as avaliações das ações assi stenciai s dirigidas ao pronto-atendimento e ao atendi mento programático de pacientes crôni cos (hipertensos e diabéti cos). A análise dessas aval iações, que apreen deram diferentes momentos consti tuintes do processo de trabal ho (objetos do trabalho, atuação dos agentes, análise dos meios de trabalho, produto do trabalho), sugerem a necessi dade da el aboração de categorias analíticas que operacionalizem a apreensão desse processo, articulando cada momento à sua totalidade. As categorias analíti cas propostas são: 1) operaci onali dade, como medida da efetivação das ações propostas; 2) efetivi dade estratégica, como medida do al cance dos resultados apreensí veis nos usuários e/ou na população, atribuíveis às ações real izadas.

Palavras-chave Hipertensão; Cuidados Primários deSaúde; Assistência Ambulatorial; Avaliação de Processos e Resultados (Cuidados de Saúde) 


\section{Introdução}

As unidades de atenção primária à saúde respondem atualmente por um grande número de consultas médicas e outros procedimentos assistenciais, representando uma importante porta de entrada para o sistema de atenção à saúde no Brasil. A esse papel assistencial, articulam-se ainda demandas sanitárias, como a vigilância e controle de doenças, bem como riscos de adoecimento, além da educação em saúde. Constituem, portanto, uma forma tecnologicamente específica de atenção que envolve síntese de saberes e complexa integração de ações individuais e coletivas, curativas e preventivas, assistenciais e educativas (Schraiber et al., 1996). Avaliar e monitorar o desempenho desses serviços é hoje uma importante necessidade para as proposições que buscam aprimorar a qualidade da atenção à saúde. Com este artigo, espera-se oferecer para a discussão e a necessária adaptação às especificidades locais uma abordagem avaliatória viável para algumas questões médico-sanitárias bastante comuns na atenção primária à saúde, contribuindo para potencializar o necessário envolvimento dos gerentes e agentes diretos do trabal ho com as questões da qualidade.

Sendo assim, este trabalho vai percorrer os seguintes objetivos:

1) contribuir para o desenvolvimento de metodologias internas e rotineiras de avaliação e monitoramento do trabalho assistencial, apropriadas às principais características tecnológicas dos programas de atenção primária;

2) propor categorias orientadoras para a avaliação e o monitoramento do processo de trabalho em programas de atenção primária.

\section{A abordagem avaliatória}

Os estudos sobre avaliação de práticas ambulatoriais admitem a presença de muitos problemas metodológicos, como, por exemplo, a difícil caracterização dos episódios de doença, grande volume de queixas maldefinidas e presença importante de condições crônicas (Palmer, 1988; Sala, 1993a). A essas dificuldades, agregam-se, na atenção primária, algumas outras, decorrentes de suas especificidades tecnológicas. Destaque-se, principalmente, a complexidade da assistência prestada na qual devem articular-se finalidades diversas, como a atenção à demanda espontânea e às condições epidemiologicamente importantes, ou ainda, à boa qualidade do cuidado técnico e à cobertu- ra adequada da população adscrita (Starfield, 1992; Nemes, 1996).

De fato, a maioria dos estudos de enfoque mais teórico, no campo da avaliação em serviços de saúde, admite várias ordens de dificuldade para os processos avaliatórios, sobretudo em serviços complexos de saúde nos quais estão articulados e hierarquizados diferentes processos de trabalho (Donabedian, 1980; Nemes, 1995; Schraiber \& Nemes, 1996). Essa rede complexa de trabalho freqüentemente funciona como uma 'caixa-preta', que pode prejudicar a validade e a utilidade da avaliação. "Abrir a caixa-preta" (Chen \& Rossi, 1987), iluminando a interação entre os componentes do trabaIho, é uma necessidade que vem sendo crescentemente aceita no campo (Nemes, 1995; Schalock, 1995; Palmer \& Hargraves, 1996).

Essa abordagem, nascida da linha de avaliação de programas sociais e sistematizada por Chen (1990) sob a denominação de theorydriven evaluation, afirma que as avaliações devem considerar as características específicas dos programas e seus valores associados, mediante uma teorização prévia acerca de suas proposições. Neste texto, adota-se essa perspectiva geral para a definição da abordagem avaliatória.

As proposições tecnológicas para a atenção primária baseadas no projeto da Ação Programática em Saúde (Schraiber, 1993; Schraiber et al., 1996) indicam, nessa linha, a constituição de práticas de avaliação orientadas a partir do processo de trabalho em saúde (Mendes-Gonçalves, 1992; Nemes, 1995; Sala et al., 1996a).

Nessa perspectiva conceitual, a aproximação intelectiva realizada pela avaliação dá-se pela análise dos vários momentos do processo de trabalho, a saber:

1) Apreensão do objeto de trabal ho considera quais questões o conjunto das atividades realmente toma como relevantes, para exercer aí sua intervenção, contrapondo-se às proposições formais que deveriam orientar o conjunto do trabalho ao que, efetivamente, recortou-se como objeto.

2) Ação dos agentes do trabalho considera o desempenho dos agentes em relação aos conteúdos previstos para seus postos de trabaIho e sua compreensão acerca das dimensões parcelares e da dimensão coletiva do trabalho.

3) Instrumentos do trabal ho considera a utilização e adequação dos mesmos aos objetos e objetivos propostos para o trabalho, sua relação com a ação dos agentes e seu papel na interação serviço-usuário.

4) Produtos do trabalho considera, primeiramente, os resultados atribuíveis às ações e 
apreensíveis nos indivíduos usuários e na população-alvo; depois, a interação estabelecida entre serviço e usuário; por fim, a constituição e reconstituição das demandas em saúde referidas aos usuários e à população. Assinale-se que tais demandas são, permanentemente, repostas como novas expectativas ao trabal ho ao mesmo tempo em que demandas anteriores estão sendo satisfeitas. É claro que o trabalho, ao satisfazer demandas, age segundo seus próprios termos (os do trabalho) e não apenas nos termos da demanda constituída. Gera-se, assim, uma dupla mão na constituição dessas demandas: uma a partir da própria dinâmica social, que determina não só a distribuição e o consumo de serviços de saúde, mas também as concepções sobre o adoecer; outra, a partir da organização do trabalho, que satisfaz demandas e necessidades segundo seus próprios termos (Mendes-Gonçalves, 1992).

É evidente a superposição e interdependência desses quatro momentos. Pode-se, por exemplo, pensar as concepções do agentes acerca das demandas dos usuários simultaneamente como instrumento e produto do trabaIho. Isso obriga a avaliação a dar conta desse conjunto articulado. Note-se que não se trata, portanto, de avaliar tal ou qual atividade, ou mesmo um conjunto de atividades eleitas por sua importância ou por sua representatividade; o objetivo é aproximar-se o mais possível do conjunto do trabalho.

Tendo por base esse quadro conceitual, realiza-se um estudo de caso, no qual se analisa o processo de avaliação realizado para algumas ações assistenciais comuns aos programas das unidades básicas de saúde: atendimento à demanda espontânea (pronto-atendimento) e atividades programáticas dirigidas a doentes hipertensos e diabéticos.

\section{Material e métodos}

O estudo foi feito pela análise de alguns processos de avaliação realizados nos últimos dez anos, no Setor de Saúde do Adulto do Centro de Saúde Escola Samuel B. Pessoa, da Faculdade de Medicina da Universidade de São Paulo (CSE) (Sala et al., 1989; Dalmaso et al., 1992; Sala et al., 1993b; Schraiber et al., 1994; Sala et al., 1996b).

O CSE situa-se no bairro do Butantã, São Paulo, e atende a uma população de, aproximadamente, 45.000 pessoas. Como as demais unidades básicas, suas ações médicas são compostas pelo atendimento à demanda espontânea, realizado por meio do pronto-atendimen- to (PA) e pelo atendimento agendado aos grupos programáticos, que, no Setor de Saúde do Adulto do CSE, é constituído pelos grupos de mulheres, idosos e adolescentes. O PA é a principal porta de entrada para a assistência, cumprindo, além de seu nível próprio de atenção (atendimento a demandas emergentes, tratamento, triagem e encaminhamento médico de queixas), o papel de captador ativo de condições que são objeto de atividades programadas (Dalmaso, 1993).

As avaliações de PA foram implementadas mediante auditorias sucessivas de prontuários com critérios explícitos (Donabedian, 1981), que analisaram o atendimento enquanto captador para as atividades programadas e resolução de demandas oriundas da procura espontânea da clientela. As avaliações envolvendo crônicos foram realizadas coletando-se dados rotineiramente do prontuário médico, constituindo, assim, análises periódicas do trabalho.

A fonte de dados utilizada por esses processos de avaliação foi o registro em prontuário. No CSE, as várias modalidades de atendimento possuem registros padronizados em fichas especial mente desenhadas (Schraiber et al., 1996). Vale assinalar que a padronização dos registros facilita enormemente o processo de avaliação por implicar, em primeiro lugar, uma relação mais estável entre os termos utilizados na linguagem escrita do registro e seus significados enquanto trabalho realizado, e, em segundo lugar, um maior grau de padronização do próprio núcleo do trabalho, ordenado em razão de prioridades estabelecidas, do objeto e dos instrumentos próprios a cada conjunto de atividades.

\section{Os processos de avaliação}

\section{Auditorias de pronto-atendimento}

As auditorias de pronto-atendimento foram selecionadas para este estudo, a fim de melhor mostrar a dinâmica entre a assistência e a avaliação. Foram realizadas três auditorias de PA, que corresponderam a três momentos distintos da organização do atendimento no setor de saúde do adulto do CSE.

Embora essas auditorias abrangessem diversos objetivos para suas análises tais como a caracterização dos atendimentos segundo alguns atributos pessoais e utilização prévia do serviço, a verificação da integração desses atendimentos com os programas, com base no desempenho dos agentes do trabalho (medido pela adequação dos registros, pelo uso apro- 
priado de normas para encaminhamento para os programas e pelo grau de realização das condutas padronizadas), ou o conhecimento dos perfis de motivos para o atendimento, diagnósticos e condutas, o presente estudo vai enfocar apenas algumas questões relativas à captação de demanda e ao modo como os profissionais desempenham essa tarefa.

A primeira auditoria (Schraiber et. al., 1994) correspondeu ao período de recém-implantação do PA. Esse período foi caracterizado pela inclusão, em maior escala, do atendimento à demanda espontânea por atendimento imediato, em uma conjuntura em que o hospital de referência (Hospital Universitário) passou a restringir esse tipo de demanda e a referenciála à unidade básica. $\mathrm{O}$ aumento de pressão de demanda, por um lado, e a necessidade de estabelecer portas de entrada para as atividades programáticas, por outro, fizeram da atividade de pronto-atendimento um arranjo original de articulação entre atendimento à demanda espontânea e organização e ordenação de ações com objetivos programáticos pré-estabelecidos.

$A$ atividade de pronto-atendimento foi implantada a partir de 1989, com a participação da enfermagem, que, num primeiro contato com o usuário, faria uma triagem inicial (mediante uma ficha de atendimento com itens padronizados) e, segundo a necessidade detectada, encaminharia para consulta médica imediata, ou agendaria atendimento em outra oportunidade. A consulta médica que se seguia à triagem inicial era realizada por médicos sanitaristas e médicos clínicos. A atenção não deveria ser dirigida apenas à queixa aguda, mas também conter uma exploração mais ampliada de outras situações que poderiam ser objeto de consultas planejadas. Ao final do atendimento, o indivíduo poderia receber alta ou ser encaminhado para alguma atividade programática, ainda em processo de recém-implantação.

Naquele momento, a avaliação buscou, centralmente, reconhecer os traços mais gerais dessa atividade e sua relação com as atividades programáticas.

Valendo-se de uma amostra de atendimentos realizados durante o período de fevereiro a maio de 1989, os dados dos atendimentos foram transcritos, por pessoal de nível operacional, para uma ficha de auditoria, a qual, em seguida, era codificada e digitada em computador.

A análise dos dados mostrou, em relação ao perfil da demanda, uma grande predominância de mulheres em idade fértil, com apenas $32 \%$ dos pacientes já em seguimento, o que evidenciava uma grande possibilidade de captação de demanda.

Quanto à integração PA-programas, verificou-se que o preenchimento completo dos campos específicos da ficha de atendimento não ocorreu em $75 \%$ dos casos.

Também ficou clara a seleção ativa da demanda através das diferenças no encaminhamento de homens e mulheres que não se encontravam em seguimento programático. Assim, dos 58 homens que se encontravam nessa situação, 28\% receberam alta após serem atendidos. Para as 167 mulheres, o número caiu para $13 \%$, pois elas foram absorvidas em diversas modalidades de atendimento, uma vez que o serviço dispunha de propostas programáticas para todas elas, o que não acontecia com os homens (Sala et al., 1989).

Em relação ao trabalho médico, além do estabelecimento de perfis de diagnósticos e condutas, verificou-se que $78 \%$ dos diagnósticos não estavam presentes na consulta imediatamente anterior do paciente, ou seja, tratava-se de diagnósticos novos ou recidivas de problemas anteriormente resolvidos.

Com base nos resultados dessa primeira auditoria, foram realizadas reuniões com toda a equipe envolvida nas ações do Setor de Saúde do Adulto, e foi feita uma reestruturação do pronto-atendimento, que ganhou um registro mais padronizado, agora com campos específicos para investigação ativa de situações pertinentes aos programas e para anotação dos encaminhamentos propostos. A participação da enfermagem foi deslocada para a pós-consulta, e, por não haver mais aquela triagem inicial, todos os indivíduos passaram a ser atendidos pelo médico.

Utilizando-se uma nova ficha de atendimento, propunha-se que o médico realizasse uma investigação ativa de situações (adolescência, envelhecimento, sintomas respiratórios, procedência de zona endêmica de esquistossomose, prevenção de câncer ginecológico, vacinação) que pudessem motivar um encaminhamento para alguma das atividades programáticas oferecidas. Após esse interrogatório e o atendimento à queixa, o profissional deveria proceder ao encaminhamento para as atividades programáticas, quando fossem pertinentes.

Operacionalizada de modo semelhante à primeira, a segunda auditoria colheu dados de todos os atendimentos realizados durante os meses de outubro e novembro de 1989 e utilizou também uma ficha para transcrição dos dados.

A auditoria pôde verificar, de modo mais acurado, o modo de aderência do profissional 
(médico, nesse caso) à anamnese ativa de questões relativas aos programas e ao cumprimento das regras para encaminhamento: revelou, em linhas gerais, que havia uma al ta proporção de atendimentos com itens de anamnese padronizada (investigação ativa de situações pertinentes aos programas) deixados em branco; nas situações em que essa anamnese, uma vez realizada, exigia encaminhamento para algum dos programas, este nem sempre ocorreu (Schraiber et al., 1994).

Uma nova reformulação do PA implicou a extensão da participação da enfermagem no atendimento, que, a partir daí, passou a realizar a maior parte daquela anamnese padronizada, deixando sob responsabilidade do médico o atendimento às queixas e os encaminhamentos programáticos que se fizessem necessários.

Uma terceira auditoria (Schraiber et al., 1994), realizada nos meses de abril e maio de 1990, utilizando a mesma metodologia da segunda, revelou que, embora a anamnese padronizada tivesse menor freqüência de itens deixados em branco, as falhas no encaminhamento para os programas aumentaram (Tabela 1). Esses últimos achados foram atribuídos a mudanças na divisão do trabalho entre médico e enfermagem.

Uma nova modificação na organização do trabal ho redefiniu, mais uma vez, a divisão de atribuições relativas ao médico e à enfermagem: ao primeiro coube, nuclearmente, o atendimento às queixas, enquanto a enfermagem permaneceu com a anamnese ativa de situações pertinentes aos programas, acrescida, agora, da responsabilidade pelo encaminhamento para uma das atividades programáticas, quando se fizesse necessário.

Tomando como ponto de análise não mais imediatamente a atuação dos agentes do trabalho, mas a efetivação de um conjunto de condutas médicas dirigidas aos pacientes com diagnóstico de hipertensão arterial, pôde-se verificar, também, como o serviço atua nos diferentes momentos de sua organização (Dalmaso et. al., 1992), conforme pode ser visto na Tabela 2.

O primeiro ponto que chama a atenção é a diferença entre os coeficientes totais (número de condutas por cem atendimentos de pacientes com diagnóstico de hipertensão), caso em que se esperaria uma maior homogeneidade entre os três períodos, uma vez que não foram modificadas as padronizações de condutas em hipertensão arterial.

No caso da hipertensão arterial, além do critério diagnóstico de duas medidas alteradas em dias diferentes, essas padronizações previam que, uma vez firmado o diagnóstico no pronto-atendimento, o médico deveria iniciar orientação dietética, introduzir diurético (se necessário) e, somente quando não obtido o efeito desejável na pressão arterial, introdução de anti-hipertensivo (evidentemente, vários usuários procuravam o serviço já com o diagnóstico firmado e em algum tratamento farmacológico). Deveria também agendar exames complementares (creatinina, glicemia, colesterol, urina I e eletrocardiograma) e uma consulta de início de seguimento programático.

Observando as auditorias comparativamente, na segunda e, principalmente, na terceira, houve uma diferença importante nos coeficientes referentes a exames, o que significaria uma implementação não uniforme da padronização durante o período considerado. Também verificou-se a queda nos coeficientes referentes a medicação diurética e anti-hipertensiva, o que poderia indicar uma opção por não se iniciar a terapêutica medicamentosa no PA; a provável contrapartida disso foi o aumento do coeficiente de orientação dietética na terceira auditoria e o encaminhamento do pacien-

\section{Tabela 1}

Distribuição percentual de encaminhamento adequado para atividades previstas para cada um dos grupos programáticos, em indivíduos em primeiro atendimento no serviço, em dois períodos de auditoria - 1989/1990.

\begin{tabular}{lcc}
\hline Grupos programáticos & 2a auditoria: \% de adequação & 3a auditoria: \% de adequação \\
\hline Idosos/Adolescentes & 64 & 44 \\
Pacientes de zona endêmica de esquistossomose & 69 & 67 \\
Sintomáticos respiratórios & 80 & 37 \\
Mulheres sem Papanicolau nos dois últimos anos & 95 & 82 \\
\hline
\end{tabular}

Fonte: Dalmaso et al., 1992.

Nota: Segunda auditoria realizada entre outubro e novembro de 1989.

Terceira auditoria realizada entre abril e maio de 1990. 
Tabela 2

Distribuição de coeficientes de condutas por cem atendimentos de pacientes com diagnóstico de hipertensão, em três momentos de auditoria.

\begin{tabular}{lccc}
\hline Condutas & 1a auditoria: coeficiente & 2a auditoria: coeficiente & 3a auditoria: coeficiente \\
\hline Avaliação saúde & 6,3 & 4,5 & 8,9 \\
Uréia/Creatinina & 18,8 & 20,5 & 22,2 \\
Glicemia & 12,5 & 15,9 & 13,3 \\
Urina I & 12,5 & 20,5 & 6,7 \\
Outros exames & - & 38,6 & 6,7 \\
Observação e acompanhamento & - & 9,1 & 8,9 \\
Diuréticos & 68,8 & 79,5 & 44,4 \\
Anti-hipertensivos & 68,8 & 36,4 & 17,8 \\
Outros medicamentos & 12,5 & 11,4 & 6,7 \\
Dieta & 18,8 & 18,2 & 37,8 \\
Outras orientações terapêuticas & - & 4,5 & 8,9 \\
Encaminhamentos & - & 2,3 & 2,2 \\
Total & 231,3 & 277,3 & 188,9 \\
\hline
\end{tabular}

Fonte: Dalmaso et. al., 1992.

N ota: Primeira auditoria realizada entre fevereiro e maio de 1989.

Segunda auditoria realizada entre outubro e novembro de 1989.

Terceira auditoria realizada entre abril e maio de 1990.

te para o seguimento programático, de acordo com a orientação do programa.

Avaliação de atividades programáticas dirigidas a doentes crônicos (hipertensão arterial e diabetes mellitus)

Essas atividades programáticas incluem o pronto-atendimento, como momento ativo de captação de demanda e como continente para situações de intercorrência clínica dos indivíduos em seguimento, e a consulta programática agendada (início de seguimento e retornos). Uma vez estabelecido o diagnóstico de hipertensão ou diabetes, o usuário deveria, sempre, ser encaminhado para o atendimento programático.

A consulta programática inicial, realizada com o preenchimento de ficha padronizada, enfoca não só a hipertensão e o diabetes, mas também os fatores de risco cardiovascular e situações relacionadas ao processo de envelhecimento (como fenômeno biossocial). Os retornos (propostos, no período estudado, em intervalo quadrimestral para os usuários com adequado controle clínico) também são organizados em ficha padronizada. A enfermagem tem participação complementar, na atividade de pós-consulta.

Essa avaliação foi realizada com base em dados rotineiramente colhidos dos prontuários, cujos bancos de dados, a partir daí consti- tuídos, prestam-se à avaliação continuada e rotineira (monitoramento) das ações realizadas em cada grupo programático.

Para a apreensão das ações realizadas, foi necessário tomar-se o usuário, valendo-se do conjunto de seus atendimentos em um dado período de tempo, como unidade de análise. As informações pertinentes a cada usuário abrangeram: 1) número de atendimentos e faltas em cada uma das modalidades agendadas (pronto-atendimento, consulta de seguimento programático, atendimento de enfermagem, atendimento em grupo etc.) e em cada uma das atividades programáticas; 2 ) presença ou ausência de alguns diagnósticos de interesse para cada grupo programático; 3) média das pressões arteriais nas consultas de pronto-atendimento antes do início do seguimento programático, e média das pressões arteriais nas consultas a partir do início do seguimento programático.

Na seqüência, apresentam-se dois processos de monitoramento envolvendo portadores de doenças crônicas.

- Primeiro monitoramento: avaliação da operação

Esse monitoramento, compreendendo dados de usuários que compareceram ao serviço no período de junho de 1990 a maio de 1991 (Sala et al., 1993b), teve como objetivos: 1) caracterizar a captação dos indivíduos a partir do pron- 
to-atendimento; 2) caracterizar a utilização das várias modalidades de atendimento, pelos portadores de hipertensão arterial e/ ou diabetes mellitus.

Trata-se do primeiro momento de avaliação desse programa, que sofreu reformulações nos dois anos anteriores, notadamente no que se refere à articulação entre pronto-atendimento e consulta agendada, e de apresentação de novos enfoques de atendimento, cujos objetivos constavam de novas fichas de atendimento.

Entre os resultados dessa avaliação periódica foi possível verificar a cobertura programática em relação à população-alvo estimada para a área geográfica de abrangência do CSE; descrever essa população-objeto das ações; caracterizar a utilização que essa população fez do serviço.

$O$ atendimento a hipertensos e diabéticos estava ao redor de, respectivamente, 24,8 por mil habitantes e 6,4 por mil habitantes, quando se considera a população com 15 anos e mais, residente na área de abrangência do Centro de Saúde. Mesmo considerando que um segmento dessa população tivesse acesso a outros serviços de assistência médica além do CSE, é provável que um grupo significativo de hipertensos e de diabéticos não estivesse sendo submetido a nenhum controle de saúde, ou nem mesmo tivesse sido objeto de diagnóstico.

Dos 3.793 pacientes atendidos no setor de atenção ao adulto, 839 apresentavam hipertensão arterial e/ ou diabetes mellitus, correspondendo a uma prevalência de $62 \%$ entre os indivíduos com cinqüenta anos e mais. A Tabela 3 mostra a distribuição desses pacientes segundo seus diagnósticos e concentração de comparecimentos, que aponta para uma utilização diferenciada do serviço por parte do grupo constituído pelos hipertensos não portadores de outra doença crônica.

Os dados da Tabela 4 mostraram, inicialmente, o volume de pacientes crônicos em cada situação diagnóstica; mostraram, também, que a maior parte desses indivíduos utiliza o atendimento programático. Apenas entre os portadores de hipertensão arterial exclusiva (sem outra doença crônico-degenerativa) observou-se uma dificuldade de captação para o atendimento programático, que é indicada pela presença de um número expressivo de hipertensos exclusivos que utilizaram apenas o pronto-atendimento (Sala et al., 1993b).

Entre os indivíduos crônicos que aderiram efetivamente ao atendimento programático, também foi possível observar diferenças significativas: uma menor concentração de consultas agendadas ( 3,4 consultas por ano) entre os hipertensos exclusivos, quando comparada à de diabéticos com e sem hipertensão associada $(5,2$ consultas por ano) ou à de hipertensos com outras doenças crônicas (4,5 consultas por ano) (Tabela 3). Se esse dado pode ser conseqüente à rotina de agendamento do serviço (trimestral) e à eventual redução no intervalo de agendamento, em razão de necessidade clínica, pode também ser decorrência de um maior volume de faltas ao agendamento, que ocorre de modo diferenciado entre os quatro grupos diagnósticos: enquanto entre os hipertensos exclusivos a média de faltas foi de 17,9 por cem consultas agendadas, entre os diabéticos, diabéticos hipertensos e hipertensos com outras doenças crônicas, a média de faltas por cem consultas foi, respectivamente, de 13,8; 12,1 e 13,1.

Assim, enquanto o agendamento espel ha, de al gum modo, a iniciativa do serviço, as faltas indicam a aderência ou a assiduidade do paciente ao atendimento a ele proposto.

- Segundo monitoramento:

eficácia no controle da pressão arterial

Essa segunda avaliação periódica, compreendendo dados de hipertensos que freqüentaram o serviço no período de janeiro de 1992 a junho de 1993, tem como objetivo central verificar a

Tabela 3

Distribuição percentual de pacientes crônicos, segundo concentração de comparecimentos em consultas agendadas e diagnóstico, em pacientes inscritos no programa até o início do período estudado.* Período de junho de 1990 a maio de 1991.

\begin{tabular}{lccccc}
\hline $\begin{array}{l}\text { Concentração de } \\
\text { comparecimentos }\end{array}$ & \multicolumn{5}{c}{ Diagnóstico** } \\
& DM & HA & HA-DM & HA EXCL & \\
\hline 1 e 2 & 20,0 & 15,2 & 8,8 & 38,3 & 25,8 \\
3 a 5 & 40,0 & 57,1 & 46,1 & 45,6 & 47,9 \\
6 e 7 & 22,9 & 18,8 & 32,4 & 13,3 & 19,1 \\
8 e mais & 17,1 & 8,9 & 12,7 & 2,8 & 7,2 \\
Total \% & 100,0 & 100,0 & 100,0 & 100,0 & 100,0 \\
Número & 35 & 112 & 102 & 248 & 497 \\
M édia & 5,2 & 4,5 & 5,2 & 3,4 & 4,2 \\
DP & 3,3 & 2,0 & 2,1 & 2,0 & 2,3 \\
\hline
\end{tabular}

Fonte: Sala et al., 1993b.

$p<0,05$

* Exclui 234 pacientes que fizeram inscrição durante o período do estudo

e 108 pacientes não inscritos.

** DM = diabetes com ou sem outra doença crônica (exclui hipertensão).

$\mathrm{HA}=$ hipertensão com outra doença crônica (exclui diabetes).

HA-DM = hipertensão e diabetes com ou sem outra doença crônica.

HA EXCL = hipertensão arterial exclusiva.

DP = desvio-padrão.

N ota: Existe diferença estatisticamente significativa entre o grupo de HA EXCL

e os demais crônicos. 
eficácia das ações programáticas na redução dos níveis de pressão arterial, desde o primeiro atendimento na unidade (em pronto-atendimento), até o seguimento programático (incluindo pelo menos duas consultas programáticas) (Sala et al., 1996b).

Utilizando, também, como unidade de análise o indivíduo-objeto das atividades programáticas, esse monitoramento permitiu mensurar a intensidade do impacto das ações na redução dos níveis de pressão arterial. Obteve-se uma média de redução de $8,8 \mathrm{mmHg}$ na pressão arterial diastólica, entre o atendimento inicial no CSE e o seguimento programático, como mostra a Tabela 5.

Para a pressão arterial sistólica, obtevese também redução significativa de níveis pressóricos, com média de redução de 17,7 $\mathrm{mmHg}$.

A análise, por meio de regressão linear múltipla, demonstrou que, entre as variáveis estudadas, a pressão inicial e a porcentagem de faltas no seguimento programático estiveram associadas de modo independente com a redução da pressão arterial sistólica e da pressão arterial diastólica. A idade esteve associada independentemente apenas com a redução da pressão arterial sistólica. A participação da idade e da porcentagem de faltas no seguimento programático revelaram que o resultado final do trabalho programático não é insensível aos diferentes modos com que as pessoas assumem o cuidado com a própria saúde (Sala et al., 1996b).

Tabela 4

Distribuição percentual de pacientes crônicos segundo situação diagnóstica e uso do serviço. Período de junho de 1990 a maio de 1991.

\begin{tabular}{lcccc}
\hline Diagnóstico* & & $\begin{array}{c}\text { Uso do serviço } \\
\text { Consulta agendada** }\end{array}$ & Pronto-atendimento exclusivo*** & Total \\
\hline Diabetes & 87,1 & 12,9 & 100,0 & 62 \\
Hipertensão & 96,6 & 3,4 & 100,0 & 177 \\
Hipertensão + Diabetes & 97,8 & 2,2 & 100,0 & 139 \\
Hipertensão excl. & 80,3 & 19,7 & 100,0 & 461 \\
Total & 87,1 & 12,9 & 100,0 & 839 \\
\hline
\end{tabular}

Fonte: Sala et al., 1993b.

* Diabetes = diabetes com ou sem outra doença crônica (exclui hipertensão).

Hipertensão = hipertensão com outra doença crônica (exclui diabetes).

Hipertensão + Diabetes = hipertensão e diabetes com ou sem outra doença crônica.

Hipertensão excl. = hipertensão arterial exclusiva.

** Consulta agendada $=$ pacientes que realizaram uma ou mais consultas médicas.

agendadas no programa, no período, com qualquer número de consultas de PA.

***Pronto-atendimento exclusivo = pacientes que realizaram apenas consultas médicas de P.A.

Tabela 5

Evolução da pressão arterial diastólica segundo pressão inicial, desde o primeiro atendimento até o seguimento programático, entre hipertensos inicialmente não controlados que se inscreveram no programa entre janeiro de 1992 e junho de 1993.

\begin{tabular}{lccrrr}
\hline $\begin{array}{l}\text { PAD inicial } \\
\text { (em mmHg) }\end{array}$ & $\begin{array}{c}\text { Piora ou redução }<5 \\
\mathrm{mmHg}\end{array}$ & $\begin{array}{c}\text { Evolução da PAD } \\
\text { Redução } \geq 5 \\
\mathrm{mmHg}\end{array}$ & $\begin{array}{c}\text { Média de redução } \\
\text { (em mmHg) }\end{array}$ & DP & $\mathrm{n}$ \\
\hline$<9 *$ & 18 & 9 & $-0,4$ & 9,6 & 27 \\
95 a 114 & 70 & 116 & 8,1 & 9,7 & 186 \\
115 & 6 & 31 & 19,2 & 13,5 & 37 \\
Total & 94 & 156 & 8,8 & 11,4 & 250
\end{tabular}

Fonte: Sala et al., 1996b.

* hipertensão arterial sistólica isolada.

Kruskal-Wallis $=40,3(2 \mathrm{GL}), p<0,0001$

$\mathrm{DP}=$ desvio-padrão. 


\section{Discussão}

Os dados apresentados neste artigo buscaram resgatar trabalhos de avaliação anteriormente realizados, no sentido de sistematizar essa experiência efetivada no que se refere à questão da avaliação, ao longo de vários anos, no Centro de Saúde Escola. Sendo assim, procura-se, primeiramente, resgatar os momentos constituintes do processo de trabalho, assim como o modo pelo qual eles permitem apreender o conjunto das ações realizadas; em seguida, delineiam-se, de modo geral, as categorias analíticas que seriam necessárias para operacionalizar a apreensão do processo de trabalho.

O objeto pode ser reconhecido sob diversas perspectivas, a depender do problema que se colocou para a avaliação, em cada momento ou em cada parcela do conjunto das práticas programáticas. Na avaliação do pronto-atendimento, o objeto significativo foi a demanda espontânea que procurou o serviço, que, no processo de atendimento, transformou-se em uma demanda potencial para as atividades programáticas. Trabalhou-se, então, com dois objetos: um clínico (atendimento ao sofrimento individual), e um programático, cuja motivação não era mais individual, mas do serviço (referida a uma dimensão coletiva). É este segundo objeto que se tornou central nas avaliações apresentadas.

Foi possível reconhecer, tanto na auditoria de PA, quanto no monitoramento de crônicos, o modo como o serviço captava, e também como deixava de captar, a demanda potencial para os programas: as dificuldades mais imediatamente relacionadas à organização das atividades (as perdas pelo uso não adequado dos instrumentos de trabalho) e as perdas pela impossibilidade de motivar o indivíduo a aderir à proposta programática.

A atuação dos agentes do trabalho pôde ser apreendida, e mostrou-se decisiva na constituição do objeto. O modo como os agentes utilizaram as fichas de atendimento e realizaram as ações revela as tensões existentes entre um trabalho centrado na atenção clínica individualizada e outro, cuja perspectiva está no seu efeito sobre o conjunto dos usuários. A análise dos agentes retoma, imediatamente, a constituição dos objetos de trabalho presentes nas ações realizadas no serviço, bem como abre a questão dos instrumentos de trabalho envolvidos na ação.

A análise dos meios de trabalho (instrumentos) ficou bastante facilitada quando se consideraram os sucessivos momentos de organização das práticas (no caso, as auditorias do pronto-atendimento) e o movimento entre uma dada disposição dos instrumentos de trabalho e as suas implicações em termos de atuação dos agentes, de possibilidades de captação de demanda e de ordenação de ações dirigidas aos usuários. Em outros termos, considerar os instrumentos de trabalho significa apreciá-los na sua articulação nos outros momentos constitutivos do processo de trabalho, notadamente com os agentes e com as ações efetivas sobre os usuários.

$\mathrm{Na}$ ação efetiva do processo de trabalho, foram consideradas duas ordens de questão: a aderência dos agentes às normas e rotinas definidas no serviço, bem como a aderência e/ ou assiduidade dos usuários às proposições de atendimento oferecidas.

A avaliação de produto do trabalho foi apreendida em sua dimensão mais imediatamente relacionada ao processo de atendimento, e aí reside sua riqueza, enquanto possibilidade de indicar quais elementos do conjunto do processo de trabalho podem ser mais ou menos favoráveis aos resultados pretendidos (a identificação da assiduidade dos hipertensos aos retornos agendados como favorável ao controle dos níveis pressóricos).

$\mathrm{Na}$ avaliação de produto do trabalho, cabe notar o fato de que o material empírico não foi apropriado para apreciar a questão da constituição de necessidades em saúde, na sua relação com o momento do cuidado em saúde.

Ao focalizar esses momentos dos processos de trabalho, mediante a avaliação de atividades parcelares, foi preciso fazê-lo de modo a reconstruir a unidade do conjunto do trabalho. Por meio da reflexão sobre os modos pelos quais foram realizadas as avaliações, é possível, agora, buscar uma sistematização de categorias analíticas que sejam comprometidas com esta reconstrução, a fim de que avaliações de trabalhos parcelares possam ser, já de início, articuladas ao conjunto.

Como categorias analíticas capazes de articular, na avaliação, os trabalhos parcelares e o trabalho coletivo na unidade básica, traçam-se duas grandes linhas de orientação referidas, respectivamente, ao processo e aos resultados do trabalho: 1 ) operacionalidade e 2 ) efetividade estratégica.

A operacionalidade éa medida da possibilidade de o trabal ho realizar-se conforme a dinâmica processual estabelecida. Analisa, portanto, a operação do modelo de organização do trabalho. No material apresentado, seus principais indicadores foram:

- captação (seleção ativa de demanda - pessoas e problemas de saúde); 
- efetivação de fluxogramas e atividades;

- efetivação das padronizações de normase condutas (médicas e não médicas).

A cobertura, embora não tivesse sido efetivamente considerada nos processo de avaliação avaliados, constitui-se, também, em um importante indicador de operacionalidade.

A efetividade estratégica é a medida do alcance dos resul tados estratégi cos apreensíveis nos usuários do serviço e/ ou na população, atribuíveis às ações realizadas e inicialmente previstos por cada um dos programas ou conjunto de atividades; seus indicadores variam conforme o objeto do trabalho. No estudo apresentado, seu indicador foi a evolução dos níveis pressóricos de hipertensos sob tratamento.

Embora o material empírico não fosse propício para outros indicadores de efetividade estratégica, poderiam ser lembrados, para outras situações de avaliação, a incidência de eclâmpsia em pacientes sob controle pré-natal; o nível de informação objetiva acerca de métodos de anticoncepção pós-atividades de divulgação; a proporção de alta por cura aos seis meses de tratamento de tuberculose; o grau de incremento na autonomia e independência no cotidiano dos idosos sob atividades grupais; o conhecimento sobre a função reprodutiva em adolescentes usuários etc.
Evidentemente, operacionalidade e efetividade estratégica foram guias de abordagem do trabalho. Na avaliação é preciso considerar, conjuntamente, os indicadores obtidos sob ambas, até porque é possível encontrar graus de incompatibilidade entre elas, como seria, por exemplo, o caso de um programa de grande operacionalidade mas de baixa efetividade estratégica, ou vice-versa.

Note-se que essas categorias não pretendem recobrir todas as dimensões envolvidas na qualidade do trabalho. Avaliações referenciadas no processo de trabalho podem sustentar outros desenvolvimentos metodológicos, como, por exemplo, a elaboração de categorias imediatamente vinculadas aos resultados do trabalho, enquanto dinâmica que se estabelece entre produto do trabalho e criação/recriação de necessidades em saúde. Neste caso, uma dimensão comunicacional (Ayres, 1994), de inter-relação entre os sujeitos envolvidos no cuidado à saúde, deveria ser considerada.

Finalmente, é necessário reiterar que a efetiva argüição da qualidade exige o entendimento do processo avaliatório como instrumento necessariamente agregado e articulado à dinâmica específica dos processos de trabaIho. Para isso, é imprescindível uma relação dinâmica, criativa e democrática entre as dimensões técnicas e gerenciais do serviço.

\section{Referências}

AYRES, J. R. C. M., 1994. Ação programática e renovação das práticas médico-sanitárias: saúde e emancipação na adolescência. Revista Saúde em Debate, 42:54-58.

CHEN, H. T. \& ROSSI, P. H., 1987.The theory-driven approach to validity. Evaluation and Program Planning, 10:95-103.

CHEN, H. T., 1990. Theory-Driven Evaluations. Newbury Park: Sage.

DALMASO, A. S. W.; SALA, A.; COHEN, D. D. \& REGO, E. F., 1992. Avaliação e Organização de Serviços de Saúde: Acompanhamento da Implantação de um Modelo Programático de Atenção à Saúde a Partir do Pronto-Atendimento (PA) - 1988 a 1990. São Paulo: Centro de Saúde Escola Samuel B. Pessoa. (mimeo.) 
DALMASO, A. S. W., 1993. O atendimento não programado: o pronto-atendimento nas ações programáticas de saúde. In: Programação em SaúdeHoje (L. B. Schraiber, org.), 2a. ed., pp. 227-243, São Paulo: Hucitec.

DONABEDIAN, A., 1980. Explorations in Quality Assessement and Monitoring. Vol. 1, Michigan: Health Administration Press.

DONABEDIAN, A., 1981. Advantages and limitations of explicit criteria for assessing the quality of health care. Milbank Memorial Fund Quartely/ Health and Society, 59:100-106.

MENDES-GONÇALVES, R. B., 1992. Práticas deSaúde: Processos de Trabal ho e Necessidades. São Paulo: Cefor/ SMS-SP/PMSP (Cadernos Cefor, série Textos ํo 1).

NEMES, M. I. B., 1995. Avaliação do Trabalho Programático na Atenção Primária à Saúde. Tese de Doutorado, São Paulo: Faculdade de Medicina, Universidade de São Paulo.

NEMES, M. I. B., 1996. A prática programática em saúde. In: Saúde do Adulto: Programas e Ações em Unidade Básica (L. B. Schraiber, M. I. B. Nemes \& R. B. Mendes-Gonçalves, orgs.), pp. 47-64, São Paulo: Hucitec.

PALMER, R. H. \& HARGRAVES, L. J., 1996. Quality measurement and improvement among primary care practitioners. An overall appraisal of results of the ambulatory care medical audit demonstration project. Medical Care, 34 (Sup.):103-113.

PALMER, R. H., 1988. The challenges and prospects for quality assessement and assurance in ambulatory care. Inquiry, 25:119-131.

SALA, A.; COHEN, D. D.; REGO, E. F. \& LITVOC, J., 1989. Avaliação do Atendimento Ambulatorial através da Auditoria dos Registros: Um Estudo em Atendimento Eventual. São Paulo: Centro de Saúde Escola Prof. Samuel B. Pessoa. (mimeo.)
SALA, A., 1993a. A avaliação de programas de saúde. In: Programação em Saúde Hoje (L. B. Schraiber, org.), 2a ed., pp. 117-138, São Paulo: Hucitec.

SALA, A.; COHEN, D. D.; DALMASO, A. S. W.; LIMA, A. M. M. \& TEIXEIRA, R. R., 1993b. Avaliação do processo de atendimento a pacientes portadores de doença crônico-degenerativa em unidade básica de saúde. Revista de Saúde Pública, 27:463471.

SALA, A.; NEMES, M. I. B. \& COHEN, D. D., 1996a. A avaliação na prática programática. In: Saúde do Adulto: Programas e Ações em Unidade Básica (L. B. Schraiber, M. I. B. Nemes \& R. B. Mendes-Gonçalves, orgs.), pp. 173-192, São Paulo: Hucitec.

SALA, A.; NEMES FILHO, A. \& ELUF NETO, J., 1996b. Avaliação da efetividade do controle da hipertensão arterial em unidade básica de saúde. Revista deSaúdePública, 30:161-167.

SCHALOCK, R. L., 1995. Outcome-Based Evaluation. New York: Plenum Press.

SCHRAIBER, L. B., 1993. Programação em Saúde Hoje. São Paulo: Hucitec.

SCHRAIBER, L. B.; NEMES, M. I. B. \& DALMASO, A. S. W., 1994. O Model o Tecnológi co da Integração das Ações em Saúde na Atenção Primária. São Paulo: Faculdade de Medicina, Universidade de São Paulo. (Relatório de Pesquisa)

SCHRAIBER, L. B.; NEMES, M. I. B. \& MENDESGONÇALVES, R. B., 1996. Saúde do Adulto: Programas e Ações em Unidades Bási cas. São Paulo: Hucitec.

SCHRAIBER, L. B. \& NEMES, M. I. B., 1996. Processo de trabalho e avaliação de serviços de saúde. Cadernos Fundap, 19:106-121.

STARFIELD, B., 1992. Primary Care. Concept, Evaluation, and Policy. Oxford: Oxford University Press. 